\title{
ПОДАВЛЕНИЕ ВЛИЯНИЯ ОГРАНИЧЕННЫХ ВНЕШНИХ ВОЗМУЩЕНИЙ В СИСТЕМЕ УПРАВЛЕНИЯ ЗАПАСАМИ ЦЕПИ ПОСТАВОК
}

\author{
Дорофеев Ю.И. ${ }^{1}$ \\ ${ }^{1}$ Национальный технический университет «Харьковский политехнический институт», Харьков
}

Copyright (C 2014 by author and the journal “Automation technological and business - processes". This work is licensed under the Creative Commons Attribution International License (CC BY). http://creativecommons.org/licenses/by/4.0/

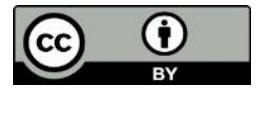

\begin{abstract}
Аннотация
Предложен подход к решению задачи построения стабилизирующего управления запасами в цепях поставок. Для подавления влияния возмущений, моделирующих изменения неизвестного, но ограниченного внешнего спроса, одновременно с обеспечением устойчивости замкнутой системы, применена методика инвариантных эллипсоидов, которая позволила сформулировать задачу в терминах линейных матричных неравенств, а синтез управления свести к последовательности задач одномерной выпуклой оптимизации и полуопределенного программирования.
\end{abstract}

Abstract

An approach to solving the problem of stabilizing inventory control synthesis for supply chains is proposed. To disturbances rejection simulating unknown but bounded external demand, while ensuring robust stability of the closed-loop system, was used the invariant ellipsoids technique, which allowed to formulate the control problem in terms of linear matrix inequalities. As a result the control synthesis problem was reduced to a sequence of onedimensional convex optimization problems and semidefinite programming.

Ключевые слова

Цепь поставок, управление запасами, метод инвариантных эллипсоидов, линейное матричное неравенство, задача полуопределенного программирования.

Введение

Цепь поставок (Supply Chain) - общеупотребительный международный термин, который обозначает совокупность взаимосвязанных бизнес-процессов, охватывающих добычу сырья, производство, хранение, транспортировку и распределение некоторой продукции с целью удовлетворения потребительского спроса.

Система управления цепями поставок (Supply Chain Management, SCM) предназначена для автоматизации и управления этапами снабжения отдельных бизнес-объектов и контроля за всеми процессами перемещения ресурсов. Главная цель управления цепями поставок - обеспечение производства и доставки необходимой продукции в нужном количестве нужному потребителю в нужное время.

Система управления запасами является одной из составляющих $S C M$, которая занимается планированием запасов и поставок с учетом выбранной модели управления запасами. Оптимальная стратегия управления запасами должна обеспечивать полное и своевременное удовлетворение внешнего спроса при условии оптимизации некоторого критерия качества работы системы. 


\section{$\underline{5}$ ТЕОРІЯ І ПРАКТИКА АВТОМАТИЗАЦІЇ БІЗНЕС-ПРОЦЕСІВ}

С точки зрения управления запасами объемы спроса на продукцию, поступающие из внешней среды, целесообразно рассматривать в качестве внешних возмущающих воздействий. Выбор модели управления запасами определяется характером спроса со стороны внешних потребителей. В настоящее время для синтеза стратегии управления запасами с заданной моделью спроса широко применяется метод прогнозирующего управления [1].

Однако, на практике, как правило, отсутствует информация для построения адекватной модели внешнего спроса, которая необходима для синтеза прогнозирующего управления. Одним из подходов к решению задачи управления запасами в условиях неопределенности спроса является использование концепции «неизвестных, но ограниченных» воздействий [2]. При этом соответствующая модель спроса характеризуется интервальной неопределенностью.

Таким образом, задача управления запасами в условиях действия неизвестного, но ограниченного спроса может рассматриваться как задача подавления влияния ограниченных внешних возмущений, которая является одной из основных в теории управления. В дискретном случае она была впервые сформулирована и для некоторых частных случаев решена в работе [3]. Для решения подобных задач применяются методы динамического программирования [4]. Ограниченные возмущения также изучались в работах, посвященных исследованию множеств достижимости [5]. В последнее десятилетие сформировался новый подход к рассматриваемой проблематике, основанный на концепции инвариантных множеств, которые широко используются в различных задачах теории автоматического управления в динамических системах при наличии неопределенностей [6]. Среди различных форм инвариантных множеств особо выделяются эллипсоиды вследствие их простой структуры и прямой связи с квадратичными функциями Ляпунова.

В рамках метода инвариантных эллипсоидов [7] в качестве технического средства используется математический аппарат линейных матричных неравенств (Linear Matrix Inequality, LMI) [8]. В настоящее время стало очевидным, что LMI представляют собой общий метод анализа и синтеза линейных систем как в непрерывном, так и в дискретном случае. Однако в большинстве работ, посвященных задаче подавления ограниченных внешних возмущений, техника LMI применяется для подавления возмущений, ограниченных в какой-либо норме. Тогда как спецификой задач управления запасами является неотрицательность значений переменных, что приводит к наличию несимметричных ограничений как на значения внешнего спроса, так и значения состояний и управляющих воздействий.

\section{Постановка задачи}

Для математического описания управляемой цепи поставок используется дискретная модель в пространстве состояний, поскольку предполагается, что получение информации о состоянии цепи и формирование управляющих воздействий происходит в дискретные моменты времени с заданным периодом дискретизации $\Delta t$. Уравнения модели описывают изменение уровня запасов каждого вида ресурсов с течением времени. В качестве переменных состояний рассматриваются наличные уровни запаса ресурсов. Управляющими воздействиями являются объемы заявок на поставку ресурсов, формируемые узлами цепи поставок в текущем периоде, а внешними возмущениями выступают объемы спроса на конечную продукцию, которые поступают извне.

Предполагается, что структура цепи поставок известна, а состояния доступны непосредственному измерению. Для описания запаздываний по управлению, обусловленных задержками в пополнении запасов относительно момента формирования заказа, используется модель дискретной задержки. Предполагается, что значения временных интервалов, определяющих длительность транспортировки и переработки ресурсов в узлах сети, известны и кратны выбранному периоду дискретизации. Тогда математическая модель управляемой цепи поставок задается разностным уравнением с запаздыванием:

$$
x(k+1)=x(k)+\sum_{t=0}^{\Lambda_{M}} B_{t} u(k-t)+E d(k),
$$

где $k=0,1, \ldots$ - номер дискретного интервала; $x(k) \in \mathbf{R}^{n}-$ вектор состояний; $u(k) \in \mathbf{R}^{m}-$ вектор управляющих воздействий; $d(k) \in \mathbf{R}^{q}$ - вектор внешних возмущений; $B_{t} \in \mathbf{R}^{n \times m}, t=\overline{0, \Lambda_{M}}$ - матрицы влияния управлений, $E \in \mathbf{R}^{n \times q}$ - матрица влияния возмущений; $\Lambda_{i} \in \mathrm{N}, i=\overline{1, n}$ - величина запаздывания управляемых потоков $i$-го узла; $\Lambda_{M}$ - максимальное значение величины запаздывания управляемых потоков между всеми парами связанных узлов цепи. Очевидно, что структура цепи поставок определяется матрицами $B_{t}$, $E$, методика построения которых изложена в работе [9].

В процессе функционирования системы должны выполняться следующие ограничения:

$$
x(k) \in X=\left\{x \in \mathbf{R}^{n}: 0 \leq x \leq x^{\max }\right\}, u(k) \in U=\left\{u \in \mathbf{R}^{m}: 0 \leq u \leq u^{\max }\right\},
$$




\section{$\underline{5}$ ТЕОРІЯ I ПРАКТИКА АВТОМАТИЗАЦІЇ БІЗНЕС-ПРОЦЕСІВ}

где векторы $x^{\max }$ и $u^{\max }$, определяющие максимальные вместимости хранилищ узлов цепи и максимальные объемы транспортировок, считаются заданными.

Будем предполагать, что векторы, описывающие внешние возмущения, удовлетворяют ограничениям:

$$
d(k) \in D=\left\{d \in \mathbf{R}^{q}: 0 \leq d^{\min } \leq d \leq d^{\max }\right\},
$$

где векторы $d^{\min }$ и $d^{\max }$ определяют граничные значения спроса и предполагаются известными.

Для системы (1) рассматривается задача синтеза стратегии управления запасами, которая для любого начального состояния $x(0) \in X$ и спроса $d(k) \in D \quad \forall k \geq 0$ обеспечивает:

1) полное и своевременное удовлетворение как внешнего, так и внутреннего спроса;

2) оптимизацию заданного критерия качества работы системы;

3) асимптотическую устойчивость замкнутой системы при ограничениях (2).

\section{Синтез стабилизирующего управления}

Первым этапом решения задачи синтеза управления является преобразование модели (1) к стандартному виду без запаздываний на основе расширения вектора состояний [10]. Вектор состояний расширенной модели будет равен $\xi(k)=\left[x^{\mathbf{T}}(k), u^{\mathbf{T}}(k-1), u^{\mathbf{T}}(k-2), \ldots, u^{\mathbf{T}}\left(k-\Lambda_{M}\right)\right]^{\mathbf{T}}$, а уравнения расширенной модели цепи поставок примут вид:

$$
\begin{aligned}
& \xi(k+1)=A \xi(k)+B u(k)+G d(k), \\
& x(k)=C \xi(k),
\end{aligned}
$$

где матрицы $A \in \mathbf{R}^{N \times N}, B \in \mathbf{R}^{N \times m}, G \in \mathbf{R}^{N \times q}, C \in \mathbf{R}^{n \times N}, N=n+m \Lambda_{M}$ имеют соответствующую блочную структуру [9]. Заметим, что вектор выхода $x(k)$ расширенной модели (3) является вектором состояний исходной модели цепи поставок (1).

Согласно теореме об аппроксимации произвольных выпуклых множеств, не обладающих свойством симметрии относительно начала координат [11], множество значений внешнего спроса $D$ может быть аппроксимировано эллипсоидом

$$
E\left(d^{*}, P_{d}\right)=\left\{d \in \mathbf{R}^{q}:\left(d(k)-d^{*}\right)^{\mathrm{T}} P_{d}^{-1}\left(d(k)-d^{*}\right) \leq 1\right\},
$$

матрица которого $P_{d}$ и вектор $d^{*}$, определяющий координаты центра, вычисляются на основании граничных значений внешнего спроса:

$$
P_{d}=\operatorname{diag}\left(\frac{q^{2}}{4}\left(d_{1}^{\max }-d_{1}^{\min }\right)^{2}, \ldots, \frac{q^{2}}{4}\left(d_{q}^{\max }-d_{q}^{\min }\right)^{2}\right), \quad d^{*}=\frac{1}{2}\left(d^{\min }+d^{\max }\right) .
$$

Будем строить закон управления в виде линейной нестационарной обратной связи по сигналу рассогласования между наличным и страховым уровнями запаса:

$$
u(k)=K(k)\left(\xi(k)-\xi^{*}\right),
$$

где $K(k) \in \mathbf{R}^{m \times N}$ - матрица коэффициентов обратной связи в момент времени $k$.

Значения вектора $\xi^{*}$, состоящего из векторов $x^{*}$ в количестве $\Lambda_{M}+1$, и определяющего размер страховых запасов, вычисляются на основании средних значений внешнего спроса с помощью продуктивной модели Леонтьева:

$$
x^{*}=(I-\Pi)^{-1} d^{\text {mean }}, \quad d_{j}^{\text {mean }}= \begin{cases}\frac{q}{2}\left(d_{j}^{\max }+d_{j}^{\min }\right), & j=\overline{1, q}, \\ 0, & j=\overline{q+1, n},\end{cases}
$$

где $\Pi$ - технологическая матрица, значение элемента $(i, j)$ которой равно количеству единиц ресурса $i$, необходимого для производства единицы ресурса $j$.

Тогда расширенную модель замкнутой системы для управления (4) представим в виде:

$$
\begin{aligned}
& \xi(k+1)=(A+B K(k))\left(\xi(k)-\xi^{*}\right)+A \xi^{*}+G\left(d(k)-d^{*}\right)+G d^{*}, \\
& x(k)=C \xi(k) .
\end{aligned}
$$




\section{$\underline{5}$ ТЕОРІЯ І ПРАКТИКА АВТОМАТИЗАЦІЇ БІЗНЕС-ПРОЦЕСІВ}

Синтез стабилизирующих алгоритмов управления, как правило, основывается на оценивании верхнего граничного значения критерия качества с помощью квадратичной функции Ляпунова, построенной на решениях системы. Запишем квадратичный критерий качества в случае бесконечного горизонта в виде:

$$
J_{\infty}(k)=\sum_{k=0}^{\infty}\left(\left(\xi(k)-\xi^{*}\right)^{\mathrm{T}} R_{\xi}\left(\xi(k)-\xi^{*}\right)+u(k)^{\mathrm{T}} R_{u} u(k)+\Delta u^{\mathrm{T}}(k) R_{\Delta} \Delta u(k)\right), \quad \Delta u(k)=u(k)-u(k-1),
$$

где $R_{\xi} \succ 0, R_{u} \succ 0, R_{\Delta} \succ 0$ - положительно определенные диагональные весовые матрицы соответствующих размерностей. Первое слагаемое в выражении (7) определяет размеры штрафов за отклонение наличных уровней запаса ресурсов от страховых, второе - стоимость производства и транспортировки ресурсов, третье - вводится для обеспечения эффекта сглаживания скачков управляющих воздействий.

Тогда задача синтеза управления сводится к решению минимаксной задачи:

$$
u(k)=\arg \min _{u(k) \in U}\left(\max _{d(k) \in E\left(d^{*}, P_{d}\right)} J_{\infty}(k)\right) .
$$

Определим квадратичную функцию Ляпунова, построенную на решениях системы (6):

$$
V\left(\xi(k)-\xi^{*}\right)=\left(\xi(k)-\xi^{*}\right)^{\mathrm{T}} P(k)\left(\xi(k)-\xi^{*}\right),
$$

где $P(k)=P^{\mathrm{T}}(k) \in \mathbf{R}^{N \times N}, P(k) \succ 0$ - симметрическая положительно определенная матрица.

Вычислим первую разность по $k$ функции Ляпунова (9) в силу системы (6) и потребуем, чтобы $\forall k \geq 0$ выполнялось неравенство, которое гарантирует асимптотическую устойчивость замкнутой системы (6):

$$
V\left(\xi(k+1)-\xi^{*}\right)-V\left(\xi(k)-\xi^{*}\right) \leq-J_{\infty}(k)
$$

Если последнее неравенство выполняется, то следуя [13], можно показать, что функция Ляпунова $V\left(\xi(k)-\xi^{*}\right)$ $\forall k \geq 0$ определяет верхнее граничное значение критерия (7):

$$
\max _{d(k) \in E\left(d^{*}, P_{d}\right)} J_{\infty}(k) \leq V\left(\xi(k)-\xi^{*}\right) .
$$

Тогда, в соответствии с (10), задача (8) эквивалентна задаче минимизации функции Ляпунова:

$$
u(k)=\arg \min _{u(k) \in U} V\left(\xi(k)-\xi^{*}\right) .
$$

В соответствии с методом инвариантных эллипсоидов [7] их можно рассматривать как характеристику влияния внешних возмущений на траектории динамической системы. Для оценки степени влияния возмущений $d(k) \in E\left(d^{*}, P_{d}\right)$ на выходы $x(k)$ удобно использовать инвариантные эллипсоиды вида

$$
E\left(\xi^{*}, P(k)\right)=\left\{\xi \in \mathbf{R}^{N}:\left(\xi-\xi^{*}\right)^{\mathrm{T}} P^{-1}(k)\left(\xi-\xi^{*}\right) \leq 1\right\},
$$

которые аппроксимируют множества достижимости замкнутой системы (6) при действии возмущений $d(k)$.

В соответствии с [12] введем матричную переменную:

$$
Q(k)=\gamma(k) P^{-1}(k),
$$

где $\gamma(k)>0$ - положительный скаляр.

Тогда задача синтеза управления заключается в вычислении в каждый дискретный момент времени $k$ матрицы коэффициентов обратной связи $K(k)$, которая стабилизирует замкнутую систему (6) и обеспечивает минимизацию функции Ляпунова (9). Соответствующий результат представлен следующей теоремой.

Т е о р е м а. Рассмотрим систему (3) с ограничениями (2) и пусть матрицы $P(k)=\gamma(k) Q^{-1}(k)$ и

$$
K(k)=Y(k) Q^{-1}(k)
$$

получены в результате решения оптимизационной задачи:

$$
\min _{Q(k), Y(k), \alpha} \gamma(k)
$$

при ограничениях на матричные переменные $Q(k)=Q^{\mathrm{T}}(k) \in \mathbf{R}^{N \times N}, \quad Y(k) \in \mathbf{R}^{m \times N}$ и скалярные параметры $\alpha, \gamma(k)$ :

$$
\alpha \geq 0, \quad \gamma(k)>0, \quad Q(k) \succ 0, \quad\left[\begin{array}{cc}
1 & \left(\xi(k)-\xi^{*}\right)^{\mathrm{T}} \\
\left(\xi(k)-\xi^{*}\right) & Q(k)
\end{array}\right] \succeq 0,
$$




\section{$\underline{5}$ ТЕОРІЯ I ПРАКТИКА АВТОМАТИЗАЦІЇ БІЗНЕС-ПРОЦЕСІВ}

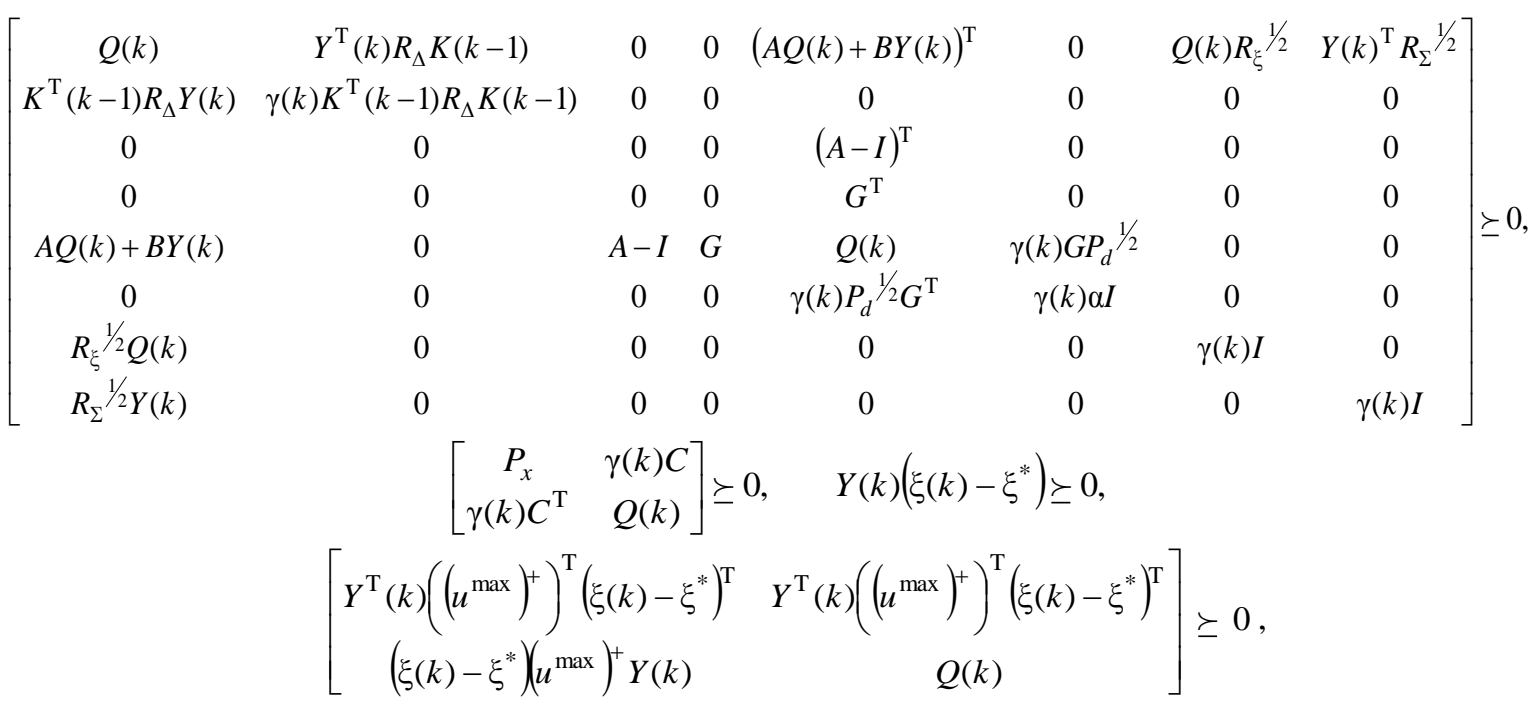

где « ${ }^{+} »-$ псевдообращение Мура-Пенроуза, $R_{\Sigma}=R_{u}+R_{\Delta}$.

Если задача (12)-(16) имеет решение, то система (3), замкнутая с помощью закона управления (4), для любого начального состояния $x(0) \in X$ и неопределенного, но ограниченного внешнего возмущения $d(k) \in E\left(d^{*}, P_{d}\right)$ является асимптотически устойчивой при ограничениях (2).

Доказательство теоремы аналогично доказательству, приведенному в работе [13].

Таким образом, задача синтеза стабилизирующего управления для цепи поставок со структурными ограничениями, подавляющего влияние интервально ограниченного внешнего спроса, сводится к задаче минимизации линейной функции (12) при ограничениях в виде линейных матричных неравенств (13)-(16), которая при фиксированном значении скаляра $\alpha$ является задачей полуопределенного программирования.

Если задача (12)-(16) имеет решение, то управляющее воздействие $u(k)$, найденное в соответствии с законом управления (4), где нестационарная матрица коэффициентов обратной связи $K(k)$ вычислена по формуле (11), применяется для управления сетью поставок в текущий момент времени. В следующий момент измеряется новое значение вектора состояний $\xi(k+1)$ и соответствующие задачи одномерной выпуклой оптимизации и полуопределенного программирования решаются в реальном времени для вычисления нового управляющего воздействия.

\section{Численный пример}

В качестве примера рассмотрим цепь поставок, которая изучалась в работе [14]. Структура цепи описывается графом $G=(\{1,2,3\},\{(2,1),(2,3),(3,1)\})$. Цепь поставок содержит $n=3$ узла: узел 1 перерабатывает продукцию узлов 2 и 3 , производит продукцию типа 1 и продает ее внешним потребителям; узел 2 получает извне сырье, производит продукцию типа 2, которую поставляет узлам 1 и 3, а также продает внешним потребителям; узел 3 из продукции узла 2 и получаемого извне сырья производит продукцию типа 3 и поставляет ее узлу 1 . Специфика рассматриваемой цепи поставок в том, что на узел 1 действует только внешний спрос; на узел 2 действует как внешний, так и внутренний спрос со стороны узлов 1 и 3 ; на узел 3 - только внутренний спрос со стороны узла 1 .

Заданы значения времени выполнения заказа в узлах сети: $T_{1}=T_{2}=2, T_{3}=1$ и времени транспортировки ресурсов между узлами сети: $T_{2,1}=T_{3,1}=T_{2,3}=1$. По формуле $\Lambda_{i}=\max \left\{T_{j, i}+T_{i}, i, j=\overline{1,3}, j \neq i\right\}$ определим величины запаздывания материальных потоков для всех узлов сети, в результате получим $\Lambda_{M}=3$.

Представим управляемые потоки $u_{1}$ и $u_{3}$ в виде гипердуг, а также добавим поток $u_{2}$, который описывает поставки сырья извне (см. рис. 1). Дуги $d_{1}, d_{2}$, изображенные пунктирными линиями, представляют внешний спрос. Значение времени транспортировки и количество единиц продукции $\Pi_{i j}$, которое требуется в соответствии с технологическим процессом, указаны для каждого управляемого потока в круглых и квадратных скобках, соответственно. Возле каждого узла в круглых скобках указаны значения времени выполнения заказа $T_{i}$. Заданы 


\section{$\underline{5}$ ТЕОРІЯ І ПРАКТИКА АВТОМАТИЗАЦІЇ БІЗНЕС-ПРОЦЕСІВ}

максимальные вместительности хранилищ цепи поставок и объемы транспортировок $x^{\max }=[100,500,100]^{T}$, $u^{\max }=[40,250,80]^{\mathrm{T}}$, граничные значения внешнего спроса $d^{\min }=[10,5]^{\mathrm{T}}, d^{\max }=[30,20]^{\mathrm{T}}$, а также начальные условия $x(0)=[100,300,100]^{\mathrm{T}}$. По формуле (7) вычислим уровни страховых запасов узлов цепи $x^{*}=[40,225,80]^{\mathrm{T}}$.

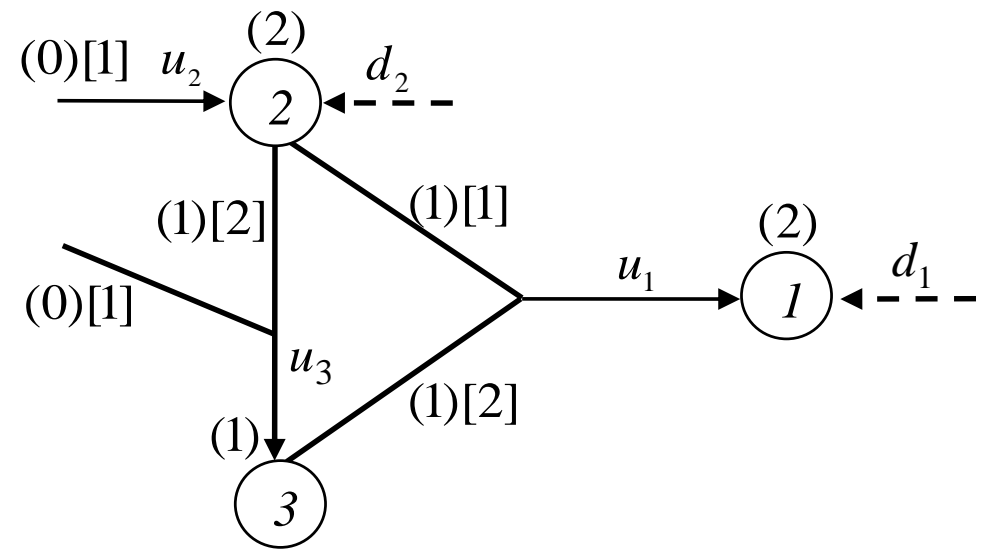

Рис. 1 - Графическое представление модели цепи поставок

Диагональные элементы весовых матриц выбраны равными $r_{\xi}=1,0 \times 10^{-8}, r_{u}=1,0 \times 10^{-7}, r_{\Delta}=9,0 \times 10^{-7}$. Численное решение задачи получено с помощью свободно распространяемого пакета CVX for MATLAB [15]. Моделирование осуществлялось в течение 15 периодов. Результаты моделирования при $\alpha=1,5 \times 10^{-2}$ и скачкообразно изменяющемся внешнем спросе для узла 1 представлены на рис. 2 , где $a-$ значения наличного и страхового уровней запасов; $\sigma$ - значения внешнего спроса и управляющих воздействий.
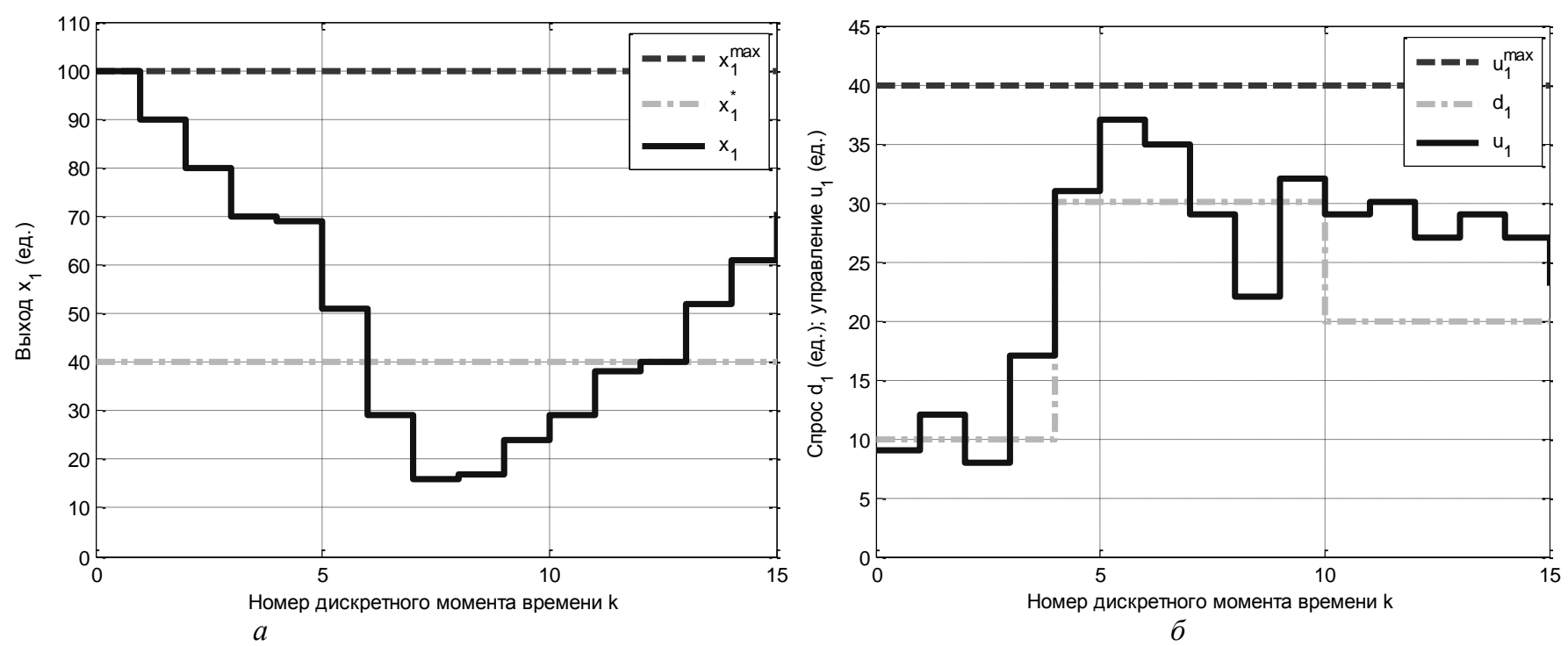

Рис. 2 - Графики переходных процессов для узла 1 цепи поставок 


\section{5 ТЕОРІЯ І ПРАКТИКА АВТОМАТИЗАЦІї БІЗНЕС-ПРОЦЕСІВ}

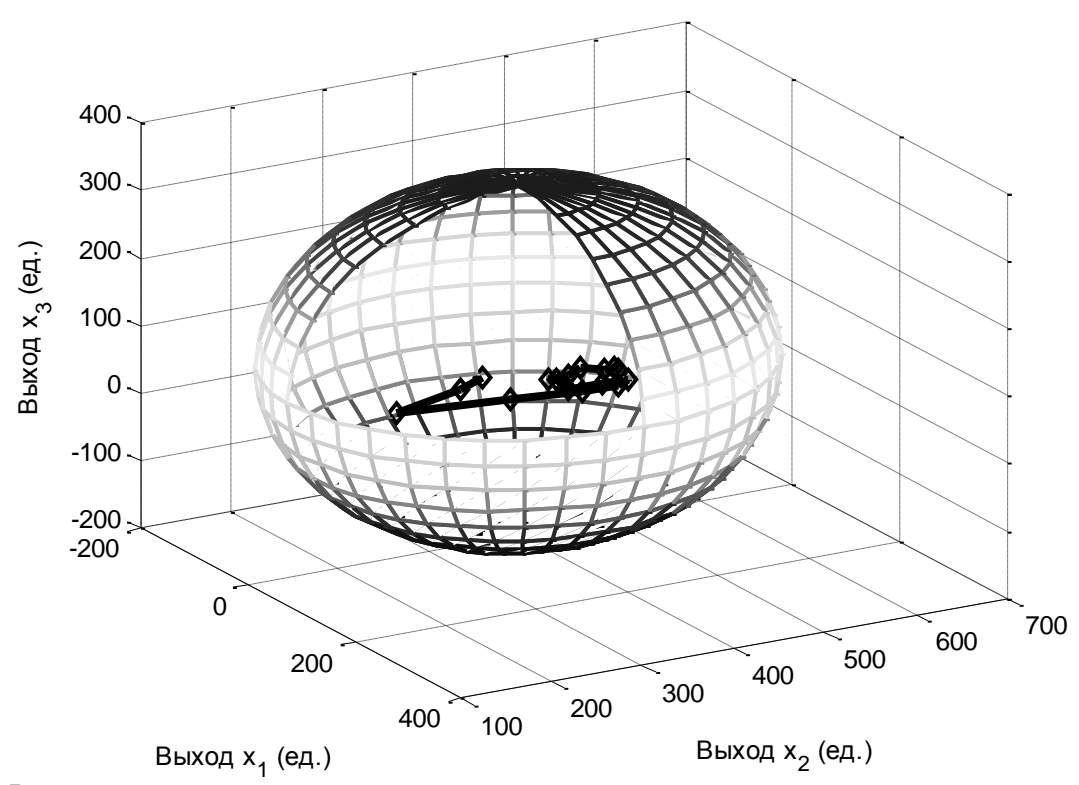

Рис. 3 - Фазовая траектория и инвариантный эллипсоид, полученный на последнем шаге

Вначале имеет место переходный процесс, длительность которого равна максимальному значению интервала запаздывания управляемых потоков в цепи $\Lambda_{M}$, обусловленный тем, что каналы транспортировки ресурсов не были загружены. Очевидно, что фазовая траектория замкнутой системы не выходит за пределы инвариантного эллипсоида, размеры которого зависят от выбранных значений весовых матриц $R_{\xi}, R_{u}$ и $R_{\Delta}$.

Результаты моделирования показали, что построенная система управления запасами обеспечивает полное и своевременное удовлетворение как внешнего, так и внутреннего спроса при минимальных затратах, связанных с производством, транспортировкой и хранением ресурсов.

\section{Выводы}

В работе предложен подход к решению задачи синтеза стабилизирующего управления запасами в цепях поставок в условиях неопределенности внешнего спроса. Для подавления влияния возмущений, моделирующих изменения неизвестного, но ограниченного спроса, одновременно с обеспечением устойчивости замкнутой системы применена методика инвариантных эллипсоидов, которая позволила сформулировать задачу в терминах линейных матричных неравенств, а синтез управления свести к задачам полуопределенного программирования и одномерной выпуклой оптимизации.

Полученное управление зависит от выбранного значения страхового уровня запасов, которое оказывает существенное влияние на величину управляющих воздействий и качество функционирования всей системы. В условиях неопределенности внешнего спроса наличие страховых запасов позволяют демпфировать его колебания и снизить риск выхода на ограничения. В рамках предложенного подхода возможен выбор оптимальных значений страховых уровней запаса ресурсов, поскольку рассмотренное решение задачи синтеза управления задает, фактически, алгоритмическую зависимость между уровнем страховых запасов и оптимальным значением критерия качества.

Предложенный подход может быть применен для решения задач синтеза робастного прогнозирующего управления запасами в цепях поставок в случае, когда уровень страхового запаса не является постоянным и для его определения используется динамическая модель, с помощью которой осуществляется прогнозирование внешнего спроса.

\section{Литература}

1. Bemporad A., Morari M. Robust model predictive control: a survey // Lecture Notes in Control and Information Sciences. - 1999. - Vol. 245. - P. 207-226;

2. Bertsekas D. P., Rhodes I. Recursive state estimation for a set-membership description of uncertainty // IEEE Trans. Automat. Control. - 1971. - Vol. 16. - P. 117-128;

3. Якубович Е. Д. Решение задачи оптимального управления для линейных дискретных систем // АиТ. 1975. - № 9. - С. 73-79.; 


\section{$\underline{5}$ ТЕОРІЯ І ПРАКТИКА АВТОМАТИЗАЦІЇ БІЗНЕС-ПРОЦЕСІВ}

4. Беллман Р., Калаба Р. Динамическое программирование и современная теория управления. - М.: Наука, 1969. - 118 c.;

5. Назин А. В., Назин С. А., Поляк Б. Т. О сходимости внешних эллипсоидальных аппроксимаций областей достижимости линейных дискретных динамических систем // АиТ. - 2004. - №8. - С. 39-61.;

6. Blanchini F., Miani S. Set Theoretic Methods in Control. - Boston: Birkhauser, 2008. - 504 p.;

7. Хлебников М. В., Поляк Б. Т., Кунцевич В. М. Оптимизация линейных систем при ограниченных внешних возмущениях (техника инвариантных эллипсоидов) // АиТ. - 2011. - № 11. - С. 9-59.;

8. Boyd S., Ghaoui E., Feron E., Balakrishnan V. Linear matrix inequalities in system and control theory. Philadelphia: SIAM, 1994. - 187 p.;

9. Дорофеев Ю. И., Никульченко А. А. Построение математических моделей управляемых сетей поставок с учетом запаздываний потоков // Системні дослідження та інформаційні технології. - 2013. - № 1.-C.16-27.;

10. Blanchini F., Pesenti R., Rinaldi F., Ukovich W. Feedback control of production-distribution systems with unknown demand and delays // IEEE Trans. on robotics and automation. - 2000. - Vol. 16. - No. 3. - P. 313-317.;

11. Черноусько Ф. Л. Оценивание фазового состояния динамических систем. Метод эллипсоидов. - М.: Наука. Физматлит, 1988. - 320 с.;

12. Kothare M. V., Balakrishnan V., Morari M. Robust constrained model predictive control using linear matrix inequalities // Automatica. - 1996. - V. 32(10). - P. 1361-1379.;

13. Дорофеев Ю. И, Любчик Л. М., Никульченко А. А. Робастное стабилизирующее управление запасами в сетях поставок в условиях неопределенности внешнего спроса и интервалов задержки пополнения запасов // Известия РАН. Теория и системы управления. - 2014. - № 5. - С.;

14. Blanchini F., Rinaldi F., Ukovich W. Least inventory control of multistorage systems with non-stochastic unknown inputs // IEEE Trans. on robotics and automation. - 1997. - Vol. 13. - P. 633-645.;

15. Grant M., Boyd S. CVX: MATLAB software for disciplined convex programming, version 2.0 beta. // URL: http://cvxr.com/cvx.

\section{References}

1. Bemporad A., Morari M. Robust model predictive control: a survey // Lecture Notes in Control and Information Sciences. - 1999. - Vol. 245. - P. 207-226.;

2. Bertsekas D. P., Rhodes I. Recursive state estimation for a set-membership description of uncertainty // IEEE Trans. Automat. Control. - 1971. - Vol. 16. - P. 117-128.;

3. Yakubovych E. D. Reshenye zadachy optymal'noho upravlenyya dlya lyneynykh dyskretnykh system // AyT. 1975. - \# 9. - S. 73-79.;

4. Bellman R., Kalaba R. Dynamycheskoe prohrammyrovanye y sovremennaya teoryya upravlenyya. - M.: Nauka, 1969. - 118 s.;

5. Nazyn A. B., Nazyn S. A., Polyak B. T. O skhodymosty vneshnykh ellypsoydal'nykh approksymatsyy oblastey dostyzhymosty lyneynykh dyskretnykh dynamycheskykh system // AyT. - 2004. - \#8. - S. 39-61.;

6. Blanchini F., Miani S. Set Theoretic Methods in Control. - Boston: Birkhauser, 2008. - 504 p.;

7. Khlebnykov M. V., Polyak B. T., Kuntsevych V. M. Optymyzatsyya lyneynykh system pry ohranychennykh vneshnykh vozmushchenyyakh (tekhnyka ynvaryantnykh ellypsoydov) // AyT. - 2011. - \# 11. - S. 9-59.;

8. Boyd S., Ghaoui E., Feron E., Balakrishnan V. Linear matrix inequalities in system and control theory. Philadelphia: SIAM, 1994. - 187 p.;

9. Dorofeev Yu. Y., Nykul'chenko A. A. Postroenye matematycheskykh modeley upravlyaemykh setey postavok s uchetom zapazdyvanyy potokov // Systemni doslidzhennya ta informatsiyni tekhnolohiyi. - 2013. - \# 1.-S.16-27.;

10. Blanchini F., Pesenti R., Rinaldi F., Ukovich W. Feedback control of production-distribution systems with unknown demand and delays // IEEE Trans. on robotics and automation. - 2000. - Vol. 16. - No. 3. - P. 313-317.;

11. Chernous'ko F. L. Otsenyvanye fazovoho sostoyanyya dynamycheskykh system. Metod ellypsoydov. - M.: Nauka. Fyzmatlyt, 1988. - 320 s.;

12. Kothare M. V., Balakrishnan V., Morari M. Robust constrained model predictive control using linear matrix inequalities // Automatica. - 1996. - V. 32(10). - P. 1361-1379.;

13. Dorofeev Yu. Y, Lyubchyk L. M., Nykul'chenko A. A. Robastnoe stabylyzyruyushchee upravlenye zapasamy v setyakh postavok v uslovyyakh neopredelennosty vneshneho sprosa y yntervalov zaderzhky popolnenyya zapasov // Yzvestyya RAN. Teoryya y systemy upravlenyya. - 2014. - \# 5. - S.;

14. Blanchini F., Rinaldi F., Ukovich W. Least inventory control of multistorage systems with non-stochastic unknown inputs // IEEE Trans. on robotics and automation. - 1997. - Vol. 13. - P. 633-645.;

15. Grant M., Boyd S. CVX: MATLAB software for disciplined convex programming, version 2.0 beta. // URL: http://cvxr.com/cvx. 